\title{
Standardization in Decentralized Economies
}

\author{
Emmanuelle Auriol \\ Michel Benaim (*)
}

This paper presents a dynamic model, inspired by evolutionary game theory, of how standards and norms emerge in decentralized economies. It shows that standardization outcomes depend on adopters' attitudes to problems caused by incompatibility. If individuals display aversion to incompatibility, standardization never fails to happen eventually, but societies sometimes end up picking inferior standards. In this case, official action can be useful to quickly achieve sensible standardization. On the other hand, when individuals display tolerance or neutrality to incompatibility, there is neither path-dependency, nor a lock-in problem, and regulation seems a poor alternative to laissez-faire.

Keywords: standardization, aversion and tolerance to incompatibility, equilibrium selection. (JEL C73, D62, L1). 


\section{Introduction}

On the threshold of the 21st century, the globalization of market economies is gathering speed with international trade growing four times as fast as the economy. In this new economic environment there is an increasing need for standardization and conformity assessment systems to come to span previously separated jurisdictions. Internationally accepted standards are necessary, first to facilitate product comparisons and reduce technical barriers to trade, ${ }^{1}$ and second to allow for better quality management and consumer protection. More than 500 organizations work with the International Organization for Standardization, ISO, on the setting of standards. ${ }^{2}$ Domestic organizations often duplicate this international activity. For instance, in the US, there are more than 700 organizations trying to promote their own, sometimes contradictory, specifications for adoption as standards. The proliferation of these organizations illustrates the importance of the standardization issue and helps to explain the difficulty of managing it successfully. In a world where technologies are constantly evolving and information about preferences is widely dispersed, setting standards is a tricky business. The consensus in the US and ISO is to rely on de facto market forces. Many in the EU, because of a political commitment to unification, favor a more centralized approach to standard setting.

This paper analyses the process of standardization in decentralized economies. It highlights the conditions under which centralized intervention is desirable and those where, on the contrary, laissez-faire seems preferable. The 
analysis is conducted in the spirit of evolutionary game theory to take account of the dynamic nature of standard adoption. Showing how adopters' attitudes toward incompatibility affect the outcome of a decentralized standardization process is the main contribution of the paper.

Aversion to incompatibility occurs if coordination failures are costly to users. This is the case with traffic regulations and signs, electrical norms, and drug formulas. People display little tolerance to incompatibility when their safety is at stake. If adopters fear standardization failures, they will attempt to avoid them. In the presence of aversion to incompatibility, we show that the decentralized adoption process inevitably leads to a single standard being established. Still, it is not always possible to predict which standard will end up being selected. If the alternatives are not very different, adopters sometimes coordinate on an inferior one. Since laissez-faire can lead to inefficient outcomes, an official intervention helps to quickly achieve sensible standardization. In every country in the world there are traffic rules, electrical norms, and other mandatory standards intended to protect users' health and safety. The European Committee for Standardization, US Consumer Product Safety Commission, and Food and Drug Administration are examples of agencies whose role is to promote such performance standards.

Neutrality to incompatibility holds when there are direct coordination externalities. This covers one-to-one communication equipment such as telephone, facsimile, and computer networks. These goods provide direct links between users. Since making a connection requires that the parties use 
compatible technologies, achieving standardization is important. More subscribers using a particular standard increases the chance of being able to communicate for all those using that standard. Under neutrality to incompatibility, we show that a decentralized standards adoption process always leads to selection of the best one. Common standards among the world's telephone, facsimile and computer Networks should come as no surprise. This standardization outcome maximizes social welfare.

Tolerance to incompatibility occurs if there are indirect coordination externalities. Examples include items like personal computers, music players, and VCRs. Here, consumers care about compatibility because the availability of complementary goods depends on sales of the primary good. As the quantity of available videos, compact disks, tapes or software increases, consumers tend to lose interest in the number of other users. Individuals then pick the technology that happens to suit them best. It should not be surprising that a decentralized adoption process occasionally results in a multiple standard equilibrium. In the presence of tolerance to incompatibility, we show that adoption of a single standard is no longer systematic, nor even necessarily optimal. Users take their time and experiment. Only the dominant technology can emerge from the standardization process as the unique choice.

From an empirical point of view, the results are consistent with the evidence on diffusion curves of new technologies, which are known to be Sshaped. The diffusion curves obtained from our theoretical analysis are also 
S-shaped. This is true regardless of individuals' attitudes towards incompatibility. Whether there is aversion, neutrality or tolerance makes no difference here. Moreover, our paper gives particular insights into the comparative benefits of decentralization and regulation in the setting of standards. When there is a public safety issue at stake, this calls for regulation or mandatory standardization. In the presence of aversion to incompatibility, official intervention is necessary to hasten coordination while preventing moves toward inferior standards. On the other hand, in the presence of tolerance or neutrality with respect to incompatibility, there is no risk of either 'lock- in' or safety hazards. This is in contrast to the risk that regulators or 'experts' might misrepresent consumers' tastes and needs. Regulation, at least in the early stages of the adoption process, seems a poor alternative to experimentation by users.

\section{Relationship with the literature}

The classic economic literature relies on non-cooperative game theory to address the standardization issue. ${ }^{3}$ This literature provides a comprehensive study of producers' behavior in the presence of network externalities, leaving the demand side of standard adoption a black box. Consumers are assumed to simultaneously adopt one of the available standards. Positive coordination externalities then generate multiple equilibria. In most cases, the authors characterize equilibria analytically, but fail to solve what Arthur (1988) calls the selection problem. In pioneering papers, Arthur $(1988,1989)$ proposes to address this issue by using stochastic models. His idea is that coordination 
externalities tend to magnify 'historical events' - the path- dependency property, and trap the dynamics in a possibly dominated standard - the lock-in property. Arthur's seminal contribution highlights the fact that, standardization generally being the result of a sequence of decentralized choices, history matters. However, he also suggests, through the examples he develops, that the alternative to regulation is a highly unpredictable, path-dependent decentralized process. This contradicts the intuitive perception that market-driven standardization is not all that unpredictable or inefficient. There remains the question of how independent, heterogeneous individuals actually coordinate their choices, that is to say, how a particular equilibrium comes to prevail.

This question lies at the core of the growing literature on evolutionary game theory and social learning, the aim of which is to study equilibrium selection in games that have multiple equilibria. This literature explains how equilibrium can emerge as a result of learning or adaptation, rather than from rational introspection. ${ }^{4}$ Kandori, Mailath and Rob (1993), in a by-now classic paper, have shown that symmetrical games (involving large numbers of myopic players and noisy mutations) naturally lead players, who hesitate between two Nash equilibria, to select the risk-dominant one. This contribution has been a major step in the area of equilibrium selection. Their result holds independently of any initial conditions or 'historical events'. More importantly, from our point of view, it has opened the door to a predictive theory of coordination problems in dynamic settings.

In the spirit of this original and fruitful line of research, this paper focuses 
on the coordination problem faced by heterogeneous individuals who have to choose between two standards. There are two time scales: a short one for individuals and a longer one for societies as a whole. The dynamic dimension is captured by the fact that individuals adopt one or the other standard sequentially and in a random order. The two time scales -individual and social- are captured by the fact that 'new-born' adopters who inherit a state of standardization from their predecessors are not forward-looking. Their limited life span requires them to optimize in the short run. One may wonder whether successive myopic adoptions will lead to some kind of unconscious collective maximizing behavior, or, on the contrary, to pure path-dependency. The answer depends on the particular standard at stake.

We show that in the long run the stochastic decentralized process of adoption behaves like a deterministic dynamic one. Since in its static version the coordination problem admits multiple equilibria, introducing the dynamics solves the selection issue. Only stable equilibria of the associated deterministic process can emerge in the long run. An important aspect of our paper is then to highlight the role concavity or convexity of the individuals' utility function plays in equilibrium selection. Contrary to the Kandori, Mailath and Rob (1993) setting, and to all other settings of which we are aware, agents' utilities are not necessarily linear with respect to the fraction of the population which chooses the same standard. We show that convexity corresponds to an aversion to incompatibility, and concavity to a tolerance for it. Linearity corresponds to neutrality. When individuals are averse to in- 
compatibility, standardization eventually occurs. The standard that ends up being selected is not always predictable, though. The fact that the equilibrium is not necessarily unique implies that the decentralized process sometimes selects the inferior standard. These are the path-dependent and lock-in properties described by Arthur (1988). On the other hand, when individuals display tolerance for incompatibility, standardization is no longer systematic. The adoption process, however, is always predictable. Finally, when individuals are neutral toward incompatibility, the decentralized process leads to standardization on the dominant standard. From the standpoint of society, this is an optimal outcome.

The paper is organized as follows. Section 2 describes the model. Section 3 presents some general results about the convergence of the stochastic process. Section 4 derives the equilibria and diffusion curves. Section 5 characterizes the optimal solution and contrasts this solution with the equilibria of section 4. Section 6 offers some concluding remarks.

\section{The Model}

We take a discrete choice model with two incompatible standards from which to choose, $A$ and $B$. Adopters have an inelastic demand for one unit of the 'good'. Their preferences are parameterized by $\delta \in[0,1]$, which we call a 'type'. The $\delta$ term can be interpreted as determining the adaptation cost incurred by agents when they have to accept a for them imperfect standard, rather than enjoying their ideal one which would have been located at $\delta$. 
That is, $\delta$ is the 'distance' between the individual and standard $A$, located at point $0,1-\delta$ being the distance between the individual and standard $B$, located at 1 . Moreover, adopters' preferences for $A$ or $B$ depend on the market share enjoyed by each standard, denoted $x_{A}$ and $x_{B}$ respectively. The preferences of a type- $\delta$ agent over standard $k(=A, B)$ are represented by the utility functions $k \rightarrow\left(1-\delta_{k}\right) u_{k}\left(x_{k}\right)$, where $\delta_{A}=\delta$ and $\delta_{B}=1-\delta$. The functions $u_{A}$ and $u_{B}$ are defined net of the price of purchasing the standard. ${ }^{5}$ It follows that a type $\delta$ agent prefers one combination of standard and market share to another if and only if it renders a higher utility to that agent:

$$
\left(A, x_{A}\right) \succeq_{\delta}\left(B, x_{B}\right) \Leftrightarrow(1-\delta) u_{A}\left(x_{A}\right) \geq \delta u_{B}\left(x_{B}\right)
$$

Functions $u_{A}$ and $u_{B}$, which are the same for all agents, but are not necessarily equal to each other, satisfy the following assumption:

A1

$$
u_{k}(0)=0 \quad \text { and } \quad u_{k}^{\prime}(x)>0 \quad \forall x \geq 0 \quad(k=A, B) .
$$

According to assumption A1, the higher the proportion of the population using a given standard, the greater the net benefit to each individual adopting it. In other words, there are positive network externalities. This assumption is consistent with the literature on learning and evolution in games. In all the papers, as in Kandori, Mailath and Rob (1993), the benefit that an agent derives from adopting a technology increases in the fraction of the rest of the population that also adopt it. However, in these papers, as well as in the network externalities literature, the payoffs are affine functions of market share. In the learning literature, where the payoff matrix is independent of players' strategies (i.e., they affect the payoff probability but not the payoff 
itself), this assumption is natural. It is less convincing in a coordination game such as standard adoption, in which individual benefits depend directly on the fraction of the population that make compatible choices. We consider any function $u_{k}$ that satisfies A1, including both concave and convex ones. ${ }^{6}$

Let $\delta_{L}^{t}$ be the marginal consumer at time $t$; i.e., the one who has no particular preference for either standard. By equation $(1), \delta_{L}^{t}$ is the solution to $(1-\delta) u_{A}\left(x_{A}^{t}\right)=\delta u_{B}\left(x_{B}^{t}\right)$, that is:

$$
\delta_{L}^{t}=\frac{u_{A}\left(x_{A}^{t}\right)}{u_{A}\left(x_{A}^{t}\right)+u_{B}\left(x_{B}^{t}\right)}
$$

We deduce that an agent $\delta$, entering the market at date $t+1$, chooses standard $A$ if $\delta \leq \delta_{L}^{t}$, and standard $B$ otherwise. This type of behavior is referred to as myopic, because the agent does not try to incorporate in his decision the way the standard will evolve in the future. In practice when consumers face a new adoption choice they heavily discount the future, in great part because they anticipate the phenomenon of obsolescence. In this paper, we take the extreme view that the adopters' psychological discount factor is infinite. This implies that, conditional on the past, their adoption choice is deterministic. ${ }^{7}$ On the contrary, the law governing their entry (the sequence of adopters) is exogenous and random. 'Nature' decides who will come next. Candidates for adoption are distributed over the interval $[0,1]$ according to a bounded density function $f($.$) whose cumulative distribution$ function is denoted $F($.$) .$

A2 $\delta$ is distributed over $[0,1]$ according to a bounded density $f($.$) .$ 
Hence, the probability that an individual entering at round $t+1$ will adopt standard $k$ is simply $P_{A}\left(x_{A}^{t}, x_{B}^{t}\right)=\operatorname{Prob}\left(\delta \leq \delta_{L}^{t}\right)$ for standard $A$, and $P_{B}\left(x_{A}^{t}, x_{B}^{t}\right)=\operatorname{Prob}\left(\delta>\delta_{L}^{t}\right)=1-P_{A}\left(x_{A}^{t}, x_{B}^{t}\right)$ for standard $B$. We deduce from assumption A2 and equation (2) that

$$
P_{A}\left(x_{A}^{t}, x_{B}^{t}\right)=F\left(\frac{u_{A}\left(x_{A}^{t}\right)}{u_{A}\left(x_{A}^{t}\right)+u_{B}\left(x_{B}^{t}\right)}\right) .
$$

It is easy to check that, under assumption A1, the probability $P_{k}^{t}=P_{k}\left(x_{A}^{t}, x_{B}^{t}\right)$ increases in the market share of standard $k$ (and thus decreases in the market share of the other standard). This is only natural, since individual decisions are shaped by positive coordination externalities. Let us now examine how successive adoption choices eventually aggregate into a collective choice.

\section{Emergence of Macro-Structure: a (Path- Dependent) Strong Law Theorem}

The dynamics of the model are as follows. Time is discrete and its horizon infinite. Period $t=1,2, \ldots$ designates the $t^{t h}$ round of adoption. The rate at which adopters arrive on the market is not important (except when looking at convergence time); the relevant time is not that of the clock, but rather that of events. At each adoption round, an agent identified by $\delta$ is randomly drawn from the population of adopters according to the probability density $f$. The sequence of adopters is exogenous and random. The adoption choice, though, is endogenous and deterministic. The agents make their choice among standards depending on their current market shares and their own particular preference. For a given individual $\delta$ at a given date $t+1$, she 
will adopt $A$ if $\delta \leq \delta_{L}^{t}$ and $B$ otherwise.

Let $N_{k}^{0} \geq 1$ be the initial number of adopters of standard $k(=A, B)$. From a technical point of view, this can be any integer larger than one. For the purpose of the analysis, though, it seems natural to focus on two cases: $N_{A}^{0}$ and $N_{B}^{0}$ both small, as would be the case in the introduction of two variants of a new technology; and $N_{A}^{0}$ small with $N_{B}^{0}$ large (or vice versa), as when a new standard begins to challenge a long-established one. Moreover, let $N_{k}^{t}$ denote the cumulative number of agents that have adopted standard $k$ at time $t$, and $N^{t}$ denote the cumulative number of adoptions which have occurred up to time $t$. Observe that $N^{t}=N_{A}^{0}+N_{B}^{0}+t$.

The market share of standard $k$ at time $t$ is $x_{k}^{t}=N_{k}^{t} / N^{t}$. Since from equation (3) $P_{k}^{t}$ denotes the probability that standard $k$ will be selected at round $t+1$ of adoption, the dynamics of market share are given by the following equation:

$$
x_{k}^{t+1}=\frac{1}{N^{t}+1} \begin{cases}N_{k}^{t}+1 & \text { with probability } P_{k}^{t} \\ N_{k}^{t} & \text { with probability } 1-P_{k}^{t} .\end{cases}
$$

Let $\alpha^{t}=\frac{1}{N^{t}+1}$. Then system (4) can be rewritten in the form:

$$
x_{k}^{t+1}= \begin{cases}\left(1-\alpha^{t}\right) x_{k}^{t}+\alpha^{t} & \text { with probability } P_{k}^{t} \\ \left(1-\alpha^{t}\right) x_{k}^{t} & \text { with probability } 1-P_{k}^{t} .\end{cases}
$$

We derive from system (5) the expected motion of market share with respect to the current state $x_{k}^{t}$. We get:

$$
E\left(x_{k}^{t+1} \mid x_{k}^{t}\right)=x_{k}^{t}+\alpha^{t}\left(P_{k}^{t}-x_{k}^{t}\right) .
$$


The interpretation of equation (6) is straightforward. The market share of standard $k$ increases in expectation if the probability of standard $k$ being adopted is greater than its current market share. Otherwise, it declines; term $\alpha^{t}\left(P_{k}^{t}-x_{k}^{t}\right)$ is positive when $P_{k}^{t}>x_{k}^{t}$ and negative when $P_{k}^{t}<x_{k}^{t}$. Indeed, if the market share of one standard is much smaller than its probability of being adopted, then this standard will be chosen on average more than proportionally to its current market share, and thus its market share will grow. By the same reasoning, if the market share of one standard is much larger than its probability of being adopted, then this standard's market share will decline. Intuition suggests that the system reaches an equilibrium when adoption probability and market share equalize. This conjecture proves to be correct.

Let $\Delta^{1}=\{(x, y) ; x \geq 0 ; y \geq 0$ and $x+y=1\}$ denote the unit simplex. The probability function $P=\left(P_{A}, P_{B}\right)$ derived from equation (3), maps the unit simplex of market shares into the unit simplex of probabilities. With the stochastic process (5), we associate the deterministic system defined on $\Delta^{1}$ (unit simplex of market share), given by the following ordinary differential equation (ODE) with dots for time derivatives:

$$
\dot{x}_{k}=P_{k}\left(x_{A}, x_{B}\right)-x_{k} \quad k=A, B .
$$

Theorem 1 establishes that the vector of market shares $x^{t}=\left(x_{A}^{t}, x_{B}^{t}\right)$ of system (5) converges towards the equilibria of the ordinary differential equation $(7)$. 
Theorem 1 (Hill-Lane-Sudderth (1980))

(i) Suppose $P: \Delta^{1} \rightarrow \Delta^{1}$, the probability function which maps the unit simplex of market shares into the unit simplex of probabilities, is continuous. Then the vector of market shares $x^{t}=\left(x_{A}^{t}, x_{B}^{t}\right)$ converges with probability one to an equilibrium $\left(x_{A}^{*}, x_{B}^{*}\right)$ of the $O D E(7)$.

(ii) Suppose $P$ maps the interior of the unit simplex into itself, and that $\left(x_{A}^{*}, x_{B}^{*}\right)$ is a stable equilibrium (as defined in the conventional way) of the ODE (7). Suppose also that the initial market share $\left(x_{A}^{0}, x_{B}^{0}\right)$ belongs to the interior of the simplex. Then the process $\left(x_{A}^{t}, x_{B}^{t}\right)$ has a positive probability of converging to $\left(x_{A}^{*}, x_{B}^{*}\right)$.

(iii) Suppose $\left(x_{A}^{*}, x_{B}^{*}\right)$ is an unstable equilibrium of the ODE (7). Then the process cannot converge to $\left(x_{A}^{*}, x_{B}^{*}\right)$ with positive probability.

Instead of studying the convergence of the stochastic process (5), it is sufficient, by virtue of Theorem 1, to study the convergence of the associated deterministic system (7). To understand this point, assume that, at the beginning of the competitive process, the initial cumulative number of subscribers, $N^{0}$, is small (the case of two new standards competing to become the market standard). Market share movement is then dominated by sharp fluctuations. When the cumulative number of subscribers is small, each new adoption makes market share jump. However, the impact of each new adoption on market share movement decreases with the number of adoptions: the first adopter has a dramatic impact, whereas the millionth one is negligible. This creates a dynamic dampening of randomness over time. ${ }^{8}$ Trapped by 
mass inertia, the stochastic process ends up being directed by the equivalent deterministic system (7). Figure 5a illustrates these fluctuations and their dampening. ${ }^{9}$ It is worth noting that, in the case of quadratic utility functions, $u_{A}(x)=a x^{2}$ and $u_{B}(y)=b y^{2}$, and uniform distribution, equation (7) gives the replicator dynamics for a homogeneous population playing a symmetrical $2 \times 2$ coordination game (i.e., with payoff: $\Pi(A, A)=a$, $\Pi(B, B)=b, \Pi(A, B)=\Pi(B, A)=0)$. That is, equation (7) becomes: $\dot{x}=x\left(a x-\left(a x^{2}+b(1-x)^{2}\right)\right)$. This paper thus offers new micro-foundation for the study of continuous-time replicator dynamics, which can be generated as the limit of a stochastic model with heterogeneous adopters in discrete time. $^{10}$

As in classic game theory, fixed points are candidates for equilibrium. However, not all fixed points can be selected as equilibria: only stable ones are eligible. An equilibrium $x_{k}^{*}$ of the ODE is locally stable if $P_{k}^{\prime}-1 \leq 0$ at $x_{k}^{*}$ (it is locally unstable otherwise). According to equation (6), if the probability of a standard being adopted in the neighborhood of an equilibrium is larger than its market share, this standard will be adopted increasingly and will diverge from the equilibrium. Symmetrically, if the probability is smaller than its market share, it will tend to converge to the equilibrium. This implies that, $x_{k}^{t}$ will diverge from a locally unstable equilibrium, and it will tend to converge to a locally stable equilibrium. ${ }^{11}$ If the deterministic system has a single stable fixed point, equilibrium is predictable. Whatever the initial conditions $N_{A}^{0}$ and $N_{B}^{0}$, the system will converge to this fixed point. 
On the other hand, if the deterministic system has multiple stable fixed points, the outcome is unpredictable due to fluctuations at the outset. In this case, equilibrium will be path-dependent. However, depending on the initial conditions, it is possible to be more precise about the results. Let $x^{*}=\left(x_{A}^{*}, x_{B}^{*}\right)$ be a stable equilibrium of $(7)$. We define the basin of attraction of $x^{*}$, denoted $B\left(x^{*}\right)$, as the set of points $x$ whose forward trajectory for the ODE is attracted by $x^{*}$. That is, $B\left(x^{*}\right)=\left\{x \in \Delta^{1} \mid \lim _{t \rightarrow \infty} x(t)=x^{*}\right\}$, where function $x(t)$ denotes the solution of equation (7) with initial condition $x(0)=x$ ( $x$ is the vector of initial market shares). The following theorem follows from Benaim (1998).

Theorem 2 Let $x^{*} \in \Delta^{1}$ be a stable equilibrium of the $O D E(7)$. Let $Q \subset$ $B\left(x^{*}\right)$ be a compact subset of the basin of attraction of $x^{*}$. There exists a positive number $k$, uniquely dependent on $Q$, such that: If, at time $\tau \geq 0$, the total number of adoptions is $N^{\tau}=N_{A}^{\tau}+N_{B}^{\tau}$, and the market share vector $\left(x_{A}^{\tau}, x_{B}^{\tau}\right)$ belongs to $Q$, then

$$
\operatorname{Prob}\left(\lim _{t \rightarrow \infty} x^{t}=x^{*}\right) \geq\left(1-\frac{2}{k N^{\tau}}\right) e^{-\frac{k}{N^{\tau}}}
$$

Theorem 2 gives a quantitative explanation of the 'mass inertia' principle described previously. If, at the $\tau$ th round of adoption, the market share is close to a stable equilibrium $x^{*}$, nothing rules out the stochastic process of adoption jumping to another basin of attraction. ${ }^{12}$ However, the larger $N^{\tau}$, the cumulative number of subscribers at time $\tau$, the smaller the probability of such a jump. 


\section{Equilibria}

To identify the standard(s) that may emerge as the long-run outcome of the stochastic process of adjustment, by virtue of Theorem 1 we need only study the fixed points of $P$. Our candidates for equilibrium are the solutions of: $P_{A}(x, 1-x)=x$ (or, equivalently, $P_{B}(x, 1-x)=1-x$ ). From equation (3) we derive:

$$
x=F\left(\frac{u_{A}(x)}{u_{A}(x)+u_{B}(1-x)}\right) .
$$

The candidates for long-run equilibrium are the pure strategy equilibria of a static coordination game. In a game where everybody adopts simultaneously, an outcome is said to be an equilibrium if no individual "would wish to defect to a different specification of the good from the one he is meant to be getting, on the (equilibrium) assumption that he would be the only defector" Farrell-Saloner (1986). It is easy to check that the pure-strategy equilibria of the static game constitute solutions to equation (8). In particular, $P_{A}(1,0)=1$ (standardization on $\left.\mathrm{A}\right)$, and $P_{A}(0,1)=0$ (standardization on B), are always equilibria. Moreover, depending on functions $u_{A}, u_{B}$, and $F$, incompatibility solutions also exist. For the purpose of analyzing these, we assume, in what follows, that the agents are uniformly distributed over $[0,1]$.

$$
\delta \text { is uniformly distributed over }[0,1] \text {. }
$$

This implies that each type of agent is equally likely to enter the market.

Under assumption A2', equation (8) simply becomes: $(1-x) u_{A}(x)=x u_{B}(1-$ $x)$. This allows us to concentrate on the impact of individuals' preferences, 
represented by $u_{A}$ and $u_{B}$, on standardization. In the spirit of the literature on uncertainty, we introduce the definitions of tolerance and aversion to incompatibility.

Definition 1 After selecting either standard $A$ or $B$, consider the choice between being matched with a population where either $(i)$ with probability $\mu$ everybody uses standard $A$ and with probability $(1-\mu)$ everybody uses standard $B$; or $(i i) \mu$ percent of the population use standard $A$ and $(1-\mu)$ percent use standard $B$. An attitude towards standardization is said to be 'incompatibility-averse' if it leads to a strict preference for $(i)$. It is said to be 'incompatibility-tolerant' if it leads to a strict preference for (ii). It is said to be 'incompatibility-neutral' otherwise.

The way coordination externalities shape preferences depends on how and why individuals coordinate. For instance, if, after choosing a vehicle with a left- or right-hand drive, the choice is to be sent to a city where either: $(i)$ with probability $1 / 2$ everybody drives on the left-hand side of the road and with probability $1 / 2$ everybody drives on the right-hand side of the road, or $(i i)$ half the people drive on the left-hand side and the other half on the right-hand side, everybody would choose $(i)$. On the other hand, when a similar choice involves desktop computer technology with Macintosh versus an IBM-compatible format, a majority of people choose (ii). Clearly, individuals' attitudes towards incompatibility depend on the nature of the standard at stake. 
Where coordination failures are very costly to users, their attitude towards standardization will be incompatibility averse. This occurs if imperfect standardization puts them at risk, as is the case with air, sea or road traffic regulations and signs, fire or electrical norms, and drug or chemical formulas. The great Baltimore fire of 1842 provides a good illustration of this. As Krislov (1997) recalls "Fire departments from the general area rushed to help and had to stand helplessly by since the couplings for their water hoses were incompatible with local designs." Typically, this is a case where standardization, even on an inferior hose design, would have been better than incompatibility. The loss of the Titanic in 1912 gives another example. The liner Californian was only a few miles away and hundreds of lives could have been saved had it picked up the Titanic's SOS call. At the first International Convention for Safety of Life at Sea (SOLAS) in London 1914, it was decided that large vessels should maintain 24-hour radio watch. This rule has remained in force ever since. By contrast, individuals' attitudes towards incompatibility will be tolerant when coordination failures cost them little. This occurs if benefits associated with a new adoption stagnate with a high number of adoptions. Examples include the video recorder, CD player, or personal computer. When the number of complementary goods (i.e., software, CDs, or videos) is large, users do not benefit from further new adoptions. From their point of view, the gains of coordination being indirect, there are decreasing returns to scale in adoption. Finally, individuals' attitudes towards incompatibility will be neutral when the marginal benefit associated with a new adoption, for a fixed population, is constant. This is the case 
with telephone, facsimile or computer networks because for one-to-one communication each subscriber is equally important. For equipment providing a direct communication service, there are constant returns to scale in adoption which characterizes neutrality.

The different attitudes towards incompatibility shape the individuals' utility functions. Tolerance to incompatibility leads, by virtue of definition 1 , to the strict choice of $(i i)$. This is equivalent to $\mu_{k} u_{k}(1)+\left(1-\mu_{k}\right) u_{k}(0)<$ $u_{k}\left(\mu_{k}\right) \forall \mu_{k} \in(0,1)$. A sufficient condition for this inequality to be true for all $\mu_{k} \in(0,1)$ is the strict concavity of $u_{k}$. In other words, concavity of $u_{k}$ indicates tolerance to incompatibility. Similarly, convexity of $u_{k}$ indicates aversion to incompatibility. Indeed, the strict convexity of $u_{k}$ implies $\mu_{k} u_{k}(1)+\left(1-\mu_{k}\right) u_{k}(0)>u_{k}\left(\mu_{k}\right) \forall \mu_{k} \in(0,1)$, which leads to the strict choice of $(i)$. Finally, neutrality to incompatibility, which leads to indifference, is equivalent to linearity: $\mu_{k} u_{k}(1)+\left(1-\mu_{k}\right) u_{k}(0)=u_{k}\left(\mu_{k}\right) \forall \mu_{k} \in(0,1)$. In what follows, we distinguish two cases: both $u_{A}($.$) and u_{B}($.$) are convex or$ linear; both $u_{A}($.$) and u_{B}($.$) are strictly concave. As will be shown, this$ difference matters.

\section{A Aversion and neutrality to incompatibility}

First of all consider the case where the utility functions are linear, that is $u_{A}(x)=a x, u_{B}(x)=b x$, and are equal to each other, $a=b$. This implies in equation (3) $P_{A}(x, 1-x)=x \forall x \in[0,1]$ : Any market share allocation $x \in[0,1]$ is a stable equilibrium of the ODE (7) and, by virtue of 
Theorem 1, a potential candidate for market equilibrium. When individuals display neutrality to incompatibility and the standards are perfect substitute, the problem is degenerated. In what follows, we rule out this case on the ground that it is non-generic. In practice there are always differences between standards. Yet if $a$ and $b$ are very close, but are not exactly equal to each other, the equilibrium is uniquely determined. ${ }^{13}$ In the linear case, we pose:

$$
a \neq b
$$

When utility functions are convex, or linear, with respect to market share, the marginal benefit associated with new adoptions increases with the number of adopters. The benefits individuals derive from standardization are large. It is intuitive that they would exceed the gains from diversity associated with an incompatibility equilibrium and eventually drive the decentralized adjustment process towards standardization.

Proposition 1 When utility functions are convex, or linear, standardization occurs with probability one. Nevertheless, the selected standard is not always predictable. It is

- either $A$ or $B$ if: $\quad u_{A}^{\prime}(0) \leq u_{B}(1)$ and $u_{B}^{\prime}(0) \leq u_{A}(1)$

$-A$ if: $\quad u_{A}^{\prime}(0)>u_{B}(1)$

$-B$ if: $\quad u_{B}^{\prime}(0)>u_{A}(1)$

Proof: Appendix A. 
When individuals display aversion or neutrality to incompatibility, there is a corner solution. The positive coordination

externalities are strong enough to push the decentralized adoption process towards standardization. The standard selected depends on the conditions illustrated in Figure 1.

\section{[Figure 1]}

The interpretation of these conditions is as follows. Condition AN1 implies that the utility associated with the adoption of standard $A$ is, for identical market shares, similar to the utility associated with the adoption of standard $B$. That is to say, $u_{A}(x)$ and $u_{B}(x)$ are not too different. When neither standard is significantly better than the other, knowledge of adopters' behavior and the characteristics of the standards are not sufficient to predict equilibrium. Depending on initial conditions and the actual play of the first rounds of adoption, either technology may end up as a de facto standard. This situation corresponds to the case described by Arthur (1988, 1989). Increasing or constant returns exacerbate 'historical events', so that, if, by chance, one of the standards has gained an adoption advantage, it increases its lead and eventually conquers the whole market. An extreme example of path-dependency, which is ruled out by assumption A3, is provided by the linear case; $A N 1$ is equivalent to $a=b .{ }^{14}$ For a nice illustration of the path-dependent and lock-in properties, see the explanation by David (1985) of how the QWERTY typewriter keyboard arrangement ended up as the US national standard. 
On the other hand, condition $A N 2$ implies that, for identical market share, the gross utility derived from the adoption of standard $A$ is always higher than that derived from the adoption of standard $B$ (and vice-versa under condition $A N 3) .{ }^{15}$ For instance, in the linear case, AN2 is equivalent to $a>b$ and $A N 3$ to $b>a$. We then say that standard $A$ has a pronounced advantage over standard $B$ (respectively standard $B$ over standard $A$ ), in the sense that it is sufficiently better to drive its competitor out of the market independently of initial conditions $\left(N_{A}^{0}, N_{B}^{0}\right)$. In the telecommunications industry, an example of such a pronounced advantage is given by the telephone as compared to the telegraph.

[Figure 2]

Figure 2 describes the probability, under the convexity assumption, that the next adopter will choose standard $A$ as a function of the current market share of $x_{A}$ (the probability of choosing $B$ as a function of $B$ 's market share is the complement in the unit square). The candidates for equilibrium (i.e., the fixed points of $P$ ) are the intersection points of function $P_{A}$ and the bisectrix. Convergence to equilibria (stable fixed points) are indicated by the arrows.

\section{B Tolerance to incompatibility}

When the utility functions are concave in market share, there are, from the individuals' point of view, decreasing returns to adoption. The marginal benefit associated with a new adoption, for a fixed population, decreases with the number of adopters. Coordination failure being less costly to users, 
it comes as no surprise that incompatibility solutions occur. 
Proposition 2 When the utility functions are strictly concave, market share is always predictable. Standardization, however, is no longer systematic. The market

- sustains incompatibility if: $\quad u_{A}^{\prime}(0) \geq u_{B}(1)$ and $u_{B}^{\prime}(0) \geq u_{A}(1)$

- standardizes on $A$ if: $\quad u_{B}^{\prime}(0)<u_{A}(1)$

- standardizes on $B$ if: $\quad u_{A}^{\prime}(0)<u_{B}(1)$

Proof: Appendix A.

Under tolerance to incompatibility, the decentralized process of adoption is always predictable. That is, concavity cushions coordination externalities and eliminates historical events, so that, given initial conditions $N_{A}^{0}$ and $N_{B}^{0}$, the outcome is known. Figure 3 illustrates that the actual outcome differs as a function of the conditions.

[Figure 3]

The interpretation of these conditions is as follows. Conditions T2 and $T 3$ are equivalent to conditions $A N 2$ and $A N 3$. When either standard has a pronounced advantage over its competitor, it eventually drives the latter out of the market and becomes the unique standard (condition T2 corresponds to a pronounced advantage of standard $A$ over standard $B$, and conversely for condition T3). In the recording industry, an example of such pronounced advantage is given by the CD as compared to vinyl.

On the other hand, under condition $T 1$, which is similar to condition $A N 1$, utility functions $u_{A}(x)$ and $u_{B}(x)$ are not too different. When neither 
standard is significantly better than the other, individuals prefer to not coordinate on a single standard rather than give up their personal preferences. Examples of such incompatibility solutions include the coexistence of Nintendo and Sega video games, Macintosh and IBM-compatible PC technology, and $\mathrm{CD}$ and cassette tape players. An indication of tolerance to incompatibility is that incompatible standards may exist side-by-side for long periods. Figure 4 illustrates the three different cases. It describes the probability that the next adopter will choose standard $A$ as a function of $A$ 's current market share. Stable equilibria are indicated by the arrows.

\section{[Figure 4]}

\section{Diffusion curves}

There is not much hard evidence on standardization. The major part of the literature is micro-oriented and qualitative in nature. An exception is found in the literature on diffusion curves for new technologies. When a new technology is introduced, it may or may not succeed in achieving a stable market share. However, when it does so, market share evolution across time is known to follow an S-shaped curve (see, for instance, Davies (1979) or Nasbeth and Ray (1974)). That is, the proportion of adoption across time is an increasing function which is at first convex and subsequently concave. In the telecommunication industry, the inflection point is often referred to as the 'critical mass' point. Telex (see Cabral and Leite (1991) for Portugal)

and telephone diffusion curves (see Hayashi (1992) for Japan) have behaved 
in this way. Since observed diffusion curves are S-shaped independently of the technology or commodity at stake, we need to compare the results of our paper with this empirical fact. With this in mind, we consider the case in which a new standard or innovation is good enough to win a stable share of the market. We compute the diffusion curve (the evolution of market share across time), in association with the deterministic system that drives the dynamic process.

Proposition 3 Diffusion curves associated with the deterministic system (7) are S-shaped independently of individuals' attitudes to incompatibility.

Proof: Appendix B.

The problem with proposition 3 is that it is established for the associated deterministic system defined by equation (7), which describes the dynamics of the process in the long run (i.e., when the cumulated number of adopters is large). A priori it says nothing about the diffusion curves of the true stochastic process that characterizes the dynamics of adoption of a new standard. The relevance of proposition 3 depends on how quickly the shape of the stochastic process defined by (5) comes to resemble the deterministic process (7).

Given an initial market share $x_{0}$, let $\{x(t)\}_{t \geq 0}$ denote the deterministic solution to (7) with initial condition $x(0)=x_{0}$ and let $\left\{x^{t}\right\}_{t=0,1, \ldots}$ denote the stochastic sequence of market shares given by (5) with the same initial 
condition. For each integer $t \geq 1$ define the new time scale

$$
\tau_{t}=1+\frac{1}{2}+\ldots+\frac{1}{t}
$$

The next theorem, which follows from Benaim (1998) section 4.2, establishes that the higher the $N^{0}$, the initial number of adopters of the old standard, the higher the probability that the diffusion curves will be S-shaped.

Theorem 3 There exist positive constants $c_{1}$ and $c_{2}$ (depending only on $u_{A}$ and $\left.u_{B}\right)$ such that: For each $\alpha \geq 0$ and every $T>0$

$$
\operatorname{Prob}\left(\sup _{t: 0 \leq \tau_{t} \leq T}\left\|x^{t}-x\left(\tau_{t}\right)\right\| \geq \alpha\right) \leq \exp \left(-N^{0} \frac{\alpha^{2}}{2 c_{1} T}+c_{2} T\right)
$$

In other words, Theorem 3 establishes that the stochastic process remains close to the deterministic path $x\left(\tau_{t}\right)$ with high probability over finite but arbitrarily large time intervals, provided the initial number of adopters $N_{0}$ is large enough. Therefore, the larger the $N_{0}$, the greater is the probability of being able to observe an S-shaped diffusion curve for the stochastic adoption process. Such behavior is observed in several numerical simulations depicted in Figure 5 (a,b,c,d). By convention the 'old' standard is $B$, the new one is $A$. The depicted market share is $x_{A}$. The time scale is $\ln (t) .{ }^{16}$ The aggregated collective behavior generated by theoretical analysis is consistent with the empirical evidences on diffusion curves.

[Figure 5] 


\section{Welfare Analysis}

As mentioned in the introduction, there is growing concern worldwide over standardization issues. Two lines of conduct emerge: the 'regulated way', which tends to favor a centralized approach to the setting of standards, and the 'decentralized way' which tends to rely on market forces. Since nonharmonized standards are technical barriers to trade, the EU favors the centralized way. Standardization in the EU is mandatory by law. ${ }^{17}$ This is in contrast with the US which favors a decentralized approach that relies on 'voluntary' standardization. The question we address next is whether there is a need for centralized intervention at all.

We compute the socially optimal market share allocation and contrast this with the decentralized equilibrium. Assuming each individual is given the same weight, the long-run optimal market share allocation maximizes consumers' average utility. For given market shares $x=\left(x_{A}, x_{B}\right)$, this average utility is:

$$
W\left(x_{A}, x_{B}\right)=\int_{0}^{\delta_{L}}(1-\delta) u_{A}\left(x_{A}\right) d \delta+\int_{\delta_{L}}^{1} \delta u_{B}\left(x_{B}\right) d \delta
$$

Integrating this expression, the long-run optimal market share allocation is the solution of:

(9) $\quad \operatorname{Max}_{x} W\left(x_{A}, x_{B}\right)=\frac{u_{A}\left(x_{A}\right)+u_{B}\left(x_{B}\right)}{2}-\frac{u_{A}\left(x_{A}\right) u_{B}\left(x_{B}\right)}{2\left[u_{A}\left(x_{A}\right)+u_{B}\left(x_{B}\right)\right]}$. 


\section{A Aversion and neutrality to incompatibility}

It is easy to check that utility function convexity implies the strict convexity of $W\left(x_{A}, x_{B}\right)=W\left(x_{A}, 1-x_{A}\right)$ in $x_{A}$. When individuals display either aversion or neutrality to incompatibility, the optimum is a corner solution: $x_{A}^{*}=1$ or $x_{A}^{*}=0$.

Proposition 4 When utility functions are convex or possibly linear, it is always optimal to standardize. If $u_{A}(1)>u_{B}(1)$, it is optimal to standardize on $A$, and, otherwise, on $B .^{18}$

This result is intuitive. Where individuals display either aversion or neutrality to incompatibility, the social gains derived from standardization on the dominant standard are very strong. Such gains overwhelm the potential diversity benefit associated with an incompatibility solution. We compare the decentralized outcome with the socially optimal solution.

Corollary 1 Under neutrality to incompatibility, the decentralized standardization process always leads to an efficient outcome. By contrast, under aversion to incompatibility, the decentralized outcome is not necessarily an optimum. It is optimal when condition AN2 or AN3 holds, but may be inefficient otherwise.

This result is straightforward. Under neutrality to incompatibility individuals always manage to standardize on the dominant technology. By virtue of proposition 4 , this is optimal. Under aversion to incompatibility, individuals 
always manage to standardize. So, when one standard performs clearly better than another one, they are able to coordinate on the superior standard. However, when the standards are not sufficiently different, as under $A N 1$, they sometimes pick the inferior one. Once this happens, it is very difficult to back-pedal or switch to the superior variant. Indeed, by virtue of Theorem 2 , the probability of switching, from a standard with 'installed base' $N$ to a standard without installed base, is proportional to $\frac{1}{N}$. The higher $N$, the lower the probability of a switch. This is illustrated by the history of the US keyboard format. All attempts made to switch from the QWERTY format to the alleged superior DVORAK one have failed (see David (1985), and discussion by Liebowitz-Margolis (1994)).

\section{B Tolerance to incompatibility}

When the utility functions are strictly concave, the results are more ambiguous. No matter how great the degree of individuals' tolerance to incompatibility (no matter how great the concavity of their utility functions), aggregate welfare itself cannot be concave. The presence of positive coordination externalities tends to convexify the problem and push the solution into the corners. So, under the following condition, which requires that the function $u_{A}$ and $u_{B}$ are weakly concave, the maximization problem is convex. There is a corner solution. ${ }^{19}$

$$
-u_{A}^{\prime \prime} u_{A}-u_{B}^{\prime \prime} u_{B} \leq\left(u_{A}^{\prime} P_{B}+u_{B}^{\prime} P_{A}\right)^{2}
$$


Similarly when one standard is totally dominated by the other (i.e., is obsolete), there is also a corner solution. Actual obsolescence is defined in the following terms. ${ }^{20}$

$\mathrm{T} 2+$ $u_{A}^{\prime}(1)>u_{B}^{\prime}(0)$ T3+ $\quad u_{B}^{\prime}(1)>u_{A}^{\prime}(0)$

However, as stated in the following proposition, the optimal trade- off between diversity and compatibility does not always lead to standardization on the dominant standard.

Proposition 5 In the concave case when either condition T2+, T3+ or T4 is satisfied, standardization on the dominant standard, argmax $\left\{u_{A}(1), u_{B}(1)\right\}$, is optimal. An incompatibility solution might dominate standardization otherwise.

Proof: Appendix C.

Concavity cushions coordination externalities so that an incompatibility solution is sometimes preferable to full blown standardization. This occurs when the benefits of adoption run out with a large number of adopters. ${ }^{21}$ Standardization deprives consumers of valuable diversity without providing corresponding compatibility benefits. We next compare the optimal solution with the decentralized outcome.

Corollary 2 Under tolerance to incompatibility, the decentralized outcome is optimal when one standard is obsolete with respect to the other, or when one standard has a pronounced advantage over the other, and, simultane- 
ously, utility functions are weakly concave. The outcome may not be efficient otherwise.

\section{Proof: Appendix D.}

When standards are not significantly different (i.e., under condition $T 1$ ), the market sustains an incompatibility equilibrium, but it is easy to find cases where it would be better to standardize. Similarly, when one standard has a pronounced advantage over the other (i.e., under T2 or T3, but not T2+ or $\mathrm{T} 3+$ ), the market always standardizes, even if, in some cases, an incompatibility solution would be better for society as a whole. An insufficient degree of standardization or excessive degree of standardization illustrates the same problem. In the presence of tolerance to incompatibility, the decentralized adoption process tends to overlook small groups of users and to favor large ones in their respective need for diversity.

\section{Regulation Vs. Laissez-Faire}

The inefficiencies involved in laissez-faire are not surprising: we are dealing with externalities. The question these inefficiencies raise, however, is whether regulation should be preferred to the decentralized process. This is a delicate question because for planners to take the optimal decision requires not only that they have a comprehensive knowledge of individuals' preference but also that they be benevolent. Especially in decentralized economies, information is widely dispersed. Users are the only ones to know their own preferences and needs. Moreover, people are generally self-interested and opportunistic. 
Institutions' attempts to assert their own authority in standardization issues in the US illustrates this opportunism problem. Most emanate from industry representatives who try to stall competition through the 'official standards' they promote. The proliferation of these organizations, which have been called a 'maze', creates a crowding- out effect. It is then left to the market to achieve standardization.

In the case of neutrality and tolerance to incompatibility, this might be a good thing, because users take time to experiment. For instance, personal computer technology was introduced in 1977 and is still not completely standardized twenty years later. The risk of 'lock-in' is irrelevant. In fact, people have been able to make the switch from traditional typewriter to computer, from vinyl record player to compact disk player, and from telegraph to telephone. This is in contrast with the risk of regulators or experts misrepresenting consumer tastes and needs. For instance, Rosenberg (1993) recalls that "Western Union was given the opportunity to purchase Bell's telephone patent in 1877 for $\$ 100,000$, but turned it down". Similarly "Marconi's invention of the 'wireless' was originally perceived as a technology for ship-to-shore communication." These examples concerning telephones and radio illustrate the fact that contemporaries were unable to foresee the future use to which these innovations would be put. Since it is difficult to forecast which innovations will be successful and which will not, the issue of experimentation by users is very important. The experience of the European Committee for Standardization (ECS) confirms that early attempts to stan- 
dardize new technologies are counterproductive. Standards that took several months to emerge were generally obsolete before their publication. The ECS has thus changed its strategy. It is now favoring a 'performance' approach to standardization; it promotes minimal standards for quality and safety rather than complete commodity design.

Whenever safety issues are at stake, thoughtful and concerted action is necessary to hasten the standardization process and orient it to the best alternative. For instance the first international standardization organization was the International Electrotechnical Commission. Created in 1906, it gave rise to the first coherent grids that turned electricity into a safer and more reliable source of energy. This was necessary to convince reluctant consumers to adopt it. In our own day, the problem of illegal drugs illustrates our point. A recent study from the National Center for Health Statistics revealed that $80 \%$ of poisoning injury death in the US are drugs related. "Opiates and cocaine were two of the leading causes of drug-related poisoning death" (May/June 98 issue of Public Health Report). Since these drugs are illegal, their composition fails to meet safety standard which explains many poisoning. Similarly, in developing countries the proliferation of counterfeit drugs, which vary in their composition from one dealer to the other, constitutes such a threat to health that a vast majority of the inhabitants prefers to avoid them. ${ }^{22}$ For instance, a survey in Zimbabwe suggests that $80 \%$ of the population rely on traditional remedies because people are too poor to buy official medicines and those available on the street are very unreliable. These problems arise be- 
cause "many developing countries lack national standards that are compatible with the international norms developed by such bodies as ISO. Moreover, the national institutions responsible for developing standards and assessing conformity are often weak" (The World Bank 1998). Without a minimal level of coercive standardization, in particular quality and safety performance standards, complex decentralized systems cannot operate. Standard setting is at the core of nation-building and a keystone of economic integration.

\section{Conclusion}

This paper offers an insight into how standards and norms emerge in decentralized economies. In particular, it provides a way to link individuals' attitudes towards incompatibility with the collective outcome of the standardization process. When individuals display aversion to incompatibility, standardization is systematic. Still, the actual standard which will be selected is not always predictable. Dispersed individuals sometimes make mistakes and pick the dominated standard. On the other hand, when individuals display tolerance to incompatibility, the decentralized process of adoption is always predictable, but it may sustain an incompatibility equilibrium and usually fails to achieve the optimal trade-off between compatibility and diversity. However, if standardization occurs, it is always on the best alternative. Finally, when individuals display neutrality to incompatibility, standardization on the dominant standard is systematic. Consistent with the empirical evidence, our analysis yields an aggregated collective behavior which generates 
S-shaped diffusion curves.

Contrary to what was suggested by Arthur's (1988) seminal work, pathdependency is not a central feature of standardization in decentralized economies. The decentralized process of adoption is predictable, except for standards that are subject to aversion to incompatibility and are not significantly different from each other. Overall predictability in this context means that, somehow, negligible and anonymous agents are able to coordinate. This is consistent with evolutionary game theory results. Successive myopic adoptions give rise to some kind of unconscious collective maximizing behavior. The emergence of this structure of collective behavior is very interesting because it is not driven by any single conscious will. It is a social phenomenon. We conclude that centralized intervention is necessary when individuals display aversion to incompatibility. In this case, coordination failures are costly to users, so that it is a priority to achieve rapid, well thought-out standardization. On the other hand, in cases of tolerance or neutrality to incompatibility, individuals take time to experiment, and never end up mistaking a bad standard for good one. Gambling on the choice of a standard, for the sake of achieving early standardization, is unlikely to improve welfare. Where there is no imperative coordination problem and in the absence of safety concerns, it seems better to rely on decentralization.

As a further extension of this study, the effect on standardization of other population probability distributions could be analyzed. It could also be extended to studying the case of competition between incompatible standards 
in the presence of a larger number of standards. 


\section{APPENDIX A: Proof of Propositions 1 and 2}

a) Equilibrium candidates: Under assumption A1, both $x_{A}=1$ and $x_{A}=0$ are solutions to equation (8). An interior solution also exists if and only if it exists $x \in] 0,1\left[\right.$ such that $H(x)=\frac{u_{A}(x)}{x}-\frac{u_{B}(1-x)}{1-x}=0$. Since $H(x)$ is increasing when $u_{A}(x)$ and $u_{B}(y)$ are convex and is decreasing when they are concave, this is equivalent to $\lim _{x \rightarrow 0} H(x)=u_{A}^{\prime}(0)-u_{B}(1)$ and $\lim _{x \rightarrow 1} H(x)=$ $u_{A}(1)-u_{B}^{\prime}(0)$ having opposite signs. In the linear case, $H(x)=a-b$, and it exists an (infinity of) interior solution if and only if $a=b$, which is ruled out by assumption A3. We easily deduce conditions $A N 1$ and $T 1$, which we complete by conditions $A N 2$ and $A N 3$, and $T 2$ and $T 3$, respectively.

b) Stability: In this one-dimensional setting an equilibrium is locally stable (respectively unstable) if the derivative of the function $G(x)=\frac{u_{A}(x)}{u_{A}(x)+u_{B}(1-x)}-$ $x$ taken at the equilibrium point is negative (respectively positive). That is, an equilibrium $x_{A}^{*}$ is locally stable (respectively unstable) if, at $x_{A}=$ $x_{A}^{*}, P_{A}^{\prime}-1<0$ (respectively $P_{A}^{\prime}-1>0$ ). It is easy to check that the equilibrium $x_{A}^{*}=0$ is stable under conditions $A N 1$ and $A N 3$ and under condition $T 2$, but unstable under condition $A N 2$ and under conditions $T 1$ and T3. Similarly it is easy to check that the equilibrium $x_{A}^{*}=1$ is stable under conditions $A N 1$ and $A N 2$ and under condition $T 3$, but unstable under condition $A N 3$ and under conditions $T 1$ and $T 2$. For the interior equilibrium $\left.x_{A}^{*}=x^{l} \in\right] 0,1\left[\left(x_{B}^{*}=1-x^{l}\right)\right.$, which exists only under condition $A N 1$ or $T 1$, we have $G^{\prime}(x)_{\mid x=x^{l}}=x^{l} \frac{\left(1-x^{l}\right) u_{A}^{\prime}\left(x^{l}\right)+x^{l} u_{B}^{\prime}\left(1-x^{l}\right)}{u_{A}\left(x^{l}\right)}-1$. We get $G^{\prime}(x)_{\mid x=x^{l}} \geq 0$ under condition $A N 1$, and $G^{\prime}(x)_{\mid x=x^{l}} \leq 0$ under condition $T 1$. The interior 
equilibrium is unstable under condition $A N 1$, and stable under condition $T 1$. These results are illustrated in figure 2 and 5 (when the probability function has a positive slope at the equilibrium point, the equilibrium is unstable; it is stable otherwise). 


\section{APPENDIX B : Proposition 3 (diffusion curves)}

Let standard $A$ be an innovation so that in the convex case condition AN2 holds, and in the concave case either condition T1 or T2 holds. the whole market, under condition $T 1$, it Let $x_{A}^{*}$ denote the market share that standard $A$ wins at equilibrium (i.e., $x_{A}^{*}=1$ under AN2 and T2, $x_{A}^{*} \in(0,1)$ under T1). Let $x(t)$ denote the diffusion curve -the market share evolution across time- of standard $A$. ¿From basically zero at its introduction time, $x(t)$ is going to rise across time and eventually converge to $x_{A}^{*}>0$. Since under AN2 (respectively, T1 and T2), $P_{A}$ is always greater than $x$ for all $0<x \leq x_{A}^{*}$ (see figures 3 and 5), we deduce from equation (7) that $x^{\prime}(t)=P_{A}(x, 1-x)-x>0$. The diffusion curve, $x(t)$, is increasing. We study next its shape. It is given by: $x^{\prime \prime}(t)=x^{\prime}(t)\left(\frac{d P_{A}(x, 1-x)}{d x}-1\right)$. Since $x^{\prime}(t)>0$, the convexity/concavity of the diffusion curve depends on the sign of $\frac{d P_{A}(x, 1-x)}{d x}-1 \forall x \leq x_{A}^{*}$. Under AN2 (respectively T1 and T2), there exists an inflection point, $\hat{t}$, for $x(t)$. It is reached when $\frac{d P_{A}(x, 1-x)}{d x}=1$. Let $\hat{x}=x(\hat{t})$ be the image of $\hat{t}$. It is the point where $P_{A}$ parallels the bisectrix, so that $\hat{x}=x(\hat{t}) \in\left(0, x_{A}^{*}\right)$. We deduce that $x^{\prime \prime}(t)>0$ for $t<\hat{t}$, and $x^{\prime \prime}(t)<0$ for $t>\hat{t}$, showing that the function, $t \rightarrow x(t)$, is $\mathrm{S}$-shaped. This is illustrated in figure 5 (the slope of $P_{A}(x, 1-x)$ is greater than the bisectrix for $x \leq \hat{x}$, and smaller for $\left.x \in\left(\hat{x}, x_{A}^{*}\right)\right)$. 


\section{APPENDIX C : Proof of Proposition 4}

Under assumption $\mathrm{T} 2+($ respectively $\mathrm{T} 3+)$, the function $\frac{u_{A}\left(x_{A}\right)+u_{B}\left(1-x_{A}\right)}{2}$ is increasing in $x_{A}$ (respectively decreasing). It is thus maximum for $x_{A}=1$ (respectively $\left.x_{A}=0\right)$. Since $W\left(x_{A}, 1-x_{A}\right)<\frac{u_{A}\left(x_{A}\right)+u_{B}\left(1-x_{A}\right)}{2}$ for all $x_{A} \in$ ]0,1[, and $W\left(x_{A}, 1-x_{A}\right)=\frac{u_{A}\left(x_{A}\right)+u_{B}\left(1-x_{A}\right)}{2}$ for $x_{A}=0$ or $x_{A}=1$, the optimum is a corner solution: $x_{A}^{*}=1$ under $\mathrm{T} 2+$, and $x_{A}^{*}=0$ under $\mathrm{T} 3+$.

When $u_{A}$ and $u_{B}$ are concave, $W\left(x_{A}, 1-x_{A}\right)$ cannot be concave. Indeed $\left.\frac{d W}{d x_{A}}\right|_{0}=\frac{-u_{B}^{\prime}(1)}{2}<0<\left.\frac{d W}{d x_{A}}\right|_{1}=\frac{u_{A}^{\prime}(1)}{2}$, where the concavity requires $\frac{d W}{d x_{A}}$ to be decreasing. It can however be convex. Let $\frac{d^{2} W}{d x_{A}{ }^{2}}=\left[\frac{u_{A}^{\prime \prime} u_{A}+u_{B}^{\prime \prime} u_{B}}{\left(u_{A}+u_{B}\right)}+\frac{\left(u_{A}^{\prime} u_{B}+u_{B}^{\prime} u_{A}\right)^{2}}{\left(u_{A}+u_{B}\right)^{3}}\right]-$ $\frac{u_{A}^{\prime \prime} u_{A}^{3}+u_{B}^{\prime \prime} u_{B}^{3}+u_{B} u_{A}\left(u_{A}^{\prime \prime} u_{A}+u_{B}^{\prime \prime} u_{B}\right)}{2\left(u_{A}+u_{B}\right)^{3}}$. Assumption T4 and $u_{k}^{\prime \prime} \leq 0$, imply that $\frac{d^{2} W}{d x_{A}{ }^{2}} \geq$ 0 . The optimum is a corner solution: $x_{A}^{*}=1$ or $x_{A}^{*}=0$. When condition T4 is not satisfied, the social welfare function can be neither convex nor concave (the problem does not admit a general analytical solution). It is then possible to find cases where standardization is no longer optimal. For instance let $u_{A}(x)=u_{B}(x)=K x-x^{2}, K>2$. It is easy to check that $W\left(\frac{1}{2}, \frac{1}{2}\right)=\frac{3}{8}(K-0.5)>W(0,1)=W(1,0)=\frac{1}{2}(K-1)$ for $K<2.5$. An incompatibility solution dominates standardization when $K \in] 2,2.5[$. 


\section{APPENDIX D : Proof of Corollary 2}

Since condition $\mathrm{T} 2+$ (respectively $\mathrm{T} 3+$ ) implies condition $\mathrm{T} 2$ (respectively T3), when $\mathrm{T} 2+$ or $\mathrm{T} 3+$ is satisfied, the market outcome (standardization) is efficient. Similarly, since under condition T4 it is optimal to standardize, when T4 holds with either $T 2$ or $T 3$ the market outcome is also efficient. However, when none of these conditions hold, the market outcome can be inefficient.

For instance, let $u_{A}(x)=u_{B}(x)=K x-x^{2}, K>2$. It is easy to check that, under this specification, condition $T 1$ is satisfied so that the market is going to sustain the incompatibility equilibrium: $x_{A}^{*}=\frac{1}{2}, x_{B}^{*}=\frac{1}{2}$. But if simultaneously $K \geq 2+\sqrt{ } 2$, condition T4 holds so that it would be socially optimal to standardize (since the left side of the inequality in T4 is minimum for $x_{k}^{t}=\frac{1}{2}$, a sufficient condition for T4 is $u^{\prime}\left(\frac{1}{2}\right)=K-1 \geq[2 x(K-x)+2(1-$ $x)(K-1+x)]^{0.5}$ which is true as soon as $\left.K \geq 2+\sqrt{ } 2\right)$. More surprisingly the market can standardize when it would be optimal to sustain diversity. Let $u_{A}(x)=u(x)$ and $u_{B}(x)=\beta u(x)$ with $u(x)=\int_{0}^{x} v(\tilde{x}) d \tilde{x}$ and

$$
v(\tilde{x})= \begin{cases}1 & \text { if } \tilde{x}<0.5-a \\ \frac{1-\epsilon}{2 a}(0.5-a-\tilde{x})+1 & \text { if } \tilde{x} \in[0.5-a, 0.5+a] \\ \epsilon & \text { if } \tilde{x}>0.5+a\end{cases}
$$

with both $a$ and $\epsilon$ positive and close to 0 . The slope of the function $u(x)$ is equal to 1 for $x<0.5-a \approx 0.5$ and is equal to $\epsilon \approx 0$ for $x>0.5+a$. Since the utility function associated to standard $k(=A, B)$ stagnates once its market share has reached the threshold of $x_{k}=\frac{1}{2}$, it would be optimal from the welfare point of view to split the market between the two standards 
when $\epsilon<\beta<\frac{u(1)}{u^{\prime}(0)} \approx \frac{1}{2}$ (or symmetrically when $\frac{1}{\epsilon}>\beta>\frac{u^{\prime}(0)}{u(1)}$ ), but despite this, standardization occurs. 


\section{REFERENCES}

Ainslie, George. "Specious Reward: A Behavioral Theory of Impulsiveness and Impulse Control", Psychological Bulletin, July 1975, $\underline{82}$ (4), pp.463-496.

Ainslie, George. "Pico-Economics", Cambridge University Press, 1992.

Auriol, Emmanuelle and Benaim Michel. "Network Externalities and Market Structure: A Dynamical Approach", 1994, mimeo GREMAQ 94.16.342 Toulouse.

Arthur, Brian W., Ermoliev Yu. M. and Kaniovsky Yu.M. "Path- Dependent Processes and the Emergence of Macro-Structure", European Journal of Operational Research, 1987, 30, pp.294-303.

Arthur, Brian W. "Self-Reinforcing Mechanisms in Economics", in The Economy as an Evolving Complex System, Addison-

Wesley Publishing Company, 1988, pp. 9-31.

Arthur, Brian W. "Competing Technologies, Increasing Returns, and Lock-in by Historical Events", The Economic Journal, March 1989, 99, pp.116-131.

Benaim, Michel. "Dynamics of Stochastic Approximations", le Séminaire de probabilités, Springer Lecture notes, 1998, (forthcoming).

Cabral, Luis M.B. and Leite Antonio P.N.. "Network Con- 
sumption Externalities, the Case of Portuguese Telex Services", in the Economics of Information Networks, Elsevier Science Publishers, 1992.

David, Paul A. "Clio and the Economics of QWERTY", American Economic Review (papers and proceedings), May 1985, 75 (2), pp. 332-337.

Davies, Stephen. The Diffusion of Process Innovations, Cambridge University Press., 1979.

Ellison, Glenn "Learning, Local Interaction, and Coordination", Econometrica, September 1993, $\underline{61}$ (5), pp.1047-1071.

Farrel, Joseph and Saloner Garth. "Standardization, Compatibility, and Innovation", Rand Journal of Economics, Spring 1985, 16 (1), pp. 70-83.

Farrel, Joseph and Saloner Garth. "Standardization and Variety", Economics Letters, 1986, 20, pp.71-74.

Foster, Dean and Young, Peyton. "Stochastic Evolutionary Game Dynamics", Theoretical Population Biology,1990, 38 (2), pp. 219-232.

Fudenberg, Drew and Kreps David M. "Learning Mixed Equilibria", Games and Economic Behavior, July 1993, $\underline{5}$ (3), 320-367.

Fudenberg, Drew and Levine David K. Theory of Learning in Game, MIT Press Series on Economic Learning and Social 
Evolution, MIT press, 1998.

Hayashi, Koichiro. "From Network Externalities to Interconnection", in the Economics of Information Networks, Elsevier Science Publishers, 1992.

Hill, Bruce M.; Lane, David and Sudderth, William. "A Strong Law for Some Generalized Urn Processes", The Annals of Probability, 1980, $\underline{8}$ (2), 214-226

Kandori, Michihiro ; Mailath George J. and Rob Rafael. "Learning, Mutation, and Long Run Equilibria in Games", Econometrica, January 1993, $\underline{61}$ (1), pp. 29- 56.

Katz, Michael L. and Shapiro Carl. "Network Externalities, Competition, and Compatibility", American Economic Review, June 1985, 75 (3), pp. 424-440.

Katz, Michael L. and Shapiro Carl. "Systems Competition and Network Effects", symposium on Network Externalities, Journal of Economic Perspectives, Spring 1994, $\underline{8}$ (2), 93-115.

Krislov, Samuel. How Nations Choose Product Standards and Standards Change Nations, University of Pittsburgh Press, 1997.

Liebowitz, S.J. and Margolis Stephen E. "Network Externalities: An Uncommon Tragedy", symposium on Network Externalities, Journal of Economic Perspectives, Spring 1994, 8 (2), pp.133-150. 
Liebowitz, S.J. and Margolis Stephen E. "Path Dependence, Lock-In, and History", Journal of Law, Economics, and Organization, 1995, 11 (1), pp.205-226.

Loewenstein, George and Prelec Drazen. "Anomaly in Intertemporal Choice: Evidence and Interpretations", Quartely

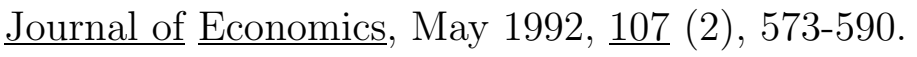

Nabseth, Lars and Ray, George-Frank The Diffusion of New Industrial Processes, Cambridge University Press, 1974.

Rosenberg, Nathan. Exploring the Black Box, chapter 11, Cambridge University Press, 1993.

Weibull, Jorgen. Evolutionary Game Theory, Cambridge, MIT Press, 1995.

World Bank. "World Development Indicators", 1998. 


\section{FOOTNOTES}

(*) Previously titled Network Externalities and Market Structure: a Dynamic Approach, first version January 1994. We are indebted to Jacques Crémer, Morris W. Hirsch, Jean- Jacques Laffont, Thomas Mariotti, Lambros Pechlivanos, Régis Renault, as well as participants in the ESEM 1994, the Networks and Competition conference, and the Fourgeaud seminar for helpful comments. This paper has also greatly benefited from two thorough referee reports and detailed and thoughtful comments of the co-editor, Preston MacAfee. We are extremely grateful for the resulting improvements. Any remaining errors are our own. Finally, we would like to thank David Liddell and John Edward Jaspers for their editorial assistance.

Emmanuelle Auriol, ARQADE and IDEI, University of Toulouse, Place Anatole-France, 31042 Toulouse cedex, France, e-mail: eauriol@cict.fr Michel Benaim, Department of Mathematics, University of Cergy-Pontoise, France.

1. Numerous studies have documented large price differences between separated markets for the same good. Among the reasons for this persistent segmentation between national markets is the existence of different product standards (World Bank (1998)).

2. The ISO standardization process, which is decentralized, involves some 2,850 technical committees and 30,000 experts. It relies on market 
forces and is based on consensus. "ISO" is not an acronym, but rather a word formed from the Greek-based prefix "iso-", meaning "equal". This has the convenient side-effect of allowing the organization to be called the same thing in all languages. (www.iso.ch (1999)).

3. Initiated by the Farrell-Saloner (1985) and Katz-Shapiro (1985) papers, the 'network externalities' literature deals with standardization issues from the standpoint of the Nash equilibrium concept. For an appraisal of this literature see the symposium 'Network Externalities' in the Journal of Economic Perspectives (1994), volume 8.

4. The founding papers of this promising literature include, among others, Foster and Young (1990), Ellison (1993), Fudenberg and Kreps (1993), Kandori, and Mailath and Rob (1993). For an up-to-date survey of the literature (and nice developments as well), see the book by Fudenberg and Levine (1998): Theory of Learning in Games.

5. Price can be either fixed or changing over time, as long as the net utility function satisfies assumption A1. This covers not only nonproprietary standards (e.g., type-writer keyboard arrangements, screw threads, bank card formats, paper sizes, rail track gauges, computer file compatibility formats such as ASCII), but also many proprietary standards. Indeed, most standardization games do not allow for multiple winners so that price competition is fierce (i.e., is of the Bertrand type). For instance, in the VHS/Beta battle over videotape format, Liebowitz-Margolis (1995) show that both technologies were always re- 
tailed at about the same price, so that price cannot be said to have played a discriminating role in consumers' decisions.

6. This paper also considers heterogeneous agents (i.e., not everyone has the same $\delta$ ), whereas Kandori, Mailath and Rob (1993) consider homogeneous agents. That is, in Kandori, Mailath and Rob (1993), the payoff of adopting strategy $k=A, B$ is for all agents $\alpha_{k}+\beta_{k} x_{k}^{t}$ with $\beta_{k}>0$. Moreover, our paper concerns network externalities among all agents, whereas interaction in Kandori, Mailath and Rob (1993) is strictly bilateral.

7. Following the work by Ainslie (1975), psychologists have emphasized 'the decline in effectiveness of rewards, as the rewards are delayed from the time of choice', thus challenging the assumption of an exponential discount function. Parametric studies and experiments have even found hyperbolic discount functions for humans and some animals. In fact, consumers are not permanent and know this, so they optimize in the short run. For a review of the literature, see Ainslie (1992) and Loewenstein-Prelec (1992). Interesting discussion of the assumption of myopic behavior can be found in Kandori, Mailath and Rob (1993) and Fudenberg-Levine (1998), Chapter 4.

8. This is in contrast to the dampening of the mutation rate in Kandori, Mailath and Rob (1993). They considers a stationary and ergodic model, in which the population is of fixed size and each agent occasionally mutates. That is, randomness about the fraction of agents 
who adopt each technology is added to the system by mutation. Hence $x_{k}^{t}$ does not converge. However, by letting the mutation rate tend to zero as a comparative statics exercise and characterizing the limit of the ergodic distribution, selection of a particular technology is ensured.

9. Figure 6 a shows the stochastic market share evolution of standard $A$ for an initial number of adopters $N_{A}^{0}=1$ and $N_{B}^{0}=20$ and utility functions $u_{A}(x)=(1+x)^{1.5}-1$ and $u_{B}(y)=y^{2}$. The final number of adoptions is 150,021 . The time scale is $\ln (t+21)$ where $t$ is the number of adoptions.

10. We are grateful to an anonymous referee for pointing out this fact. For more on replicator dynamics, see Weibull (1995) and FudenbergLevine (1998).

11. We are grateful to an anonymous referee for suggesting this discussion on stability.

12. Indeed, according to assertion (ii) of theorem 1 , this event has a positive probability of occurrence.

13. Standardization occurs on $A$ if $a>b$, and on $B$ if $a<b$.

14. When $a=b$, depending on 'historical events' any market share allocation may end up as the equilibrium. This degenerated case is ruled out by assumption A3. In the linear case, we consider either $a>b(A N 2)$ or $a<b(A N 2)$. 
15. Since the utility functions are rising and convex, AN2 (resp. AN3) implies that the line connecting $(0,0)$ to $\left(1, u_{A}^{\prime}(0)\right)$ (resp. $(0,0)$ to $\left.\left(1, u_{B}^{\prime}(0)\right)\right)$ is always below the curve $u_{A}(x)$ (resp. $\left.u_{B}(x)\right)$, see Figure 1 . Moreover, since $u_{B}(1) \geq u_{B}(x) \forall x \in[0,1], A N 2$ (resp. AN3) implies that $\left.\left.u_{A}(x)>u_{B}(x) \forall x \in\right] 0,1\right]\left(\operatorname{resp} . u_{B}(x)>u_{A}(x)\right)$.

16. $\tau_{t}=1+\frac{1}{2}+\ldots+\frac{1}{t}$ is converging to $\ln (t)$.

17. The European Committee for Standardization (ECS) publishes European Standards, which are adopted after a vote where $71 \%$ or more of the expressed votes are in favor. In contrast to other institutions, in particular the International Organization for Standardization (ISO), countries voting against are still obliged to implement European Standards by giving them the status of national standard (for more on ISO, and for links to ECS and other standardization organizations, see the ISO home-page: www.iso.ch).

18. It might be the case that $u_{A}(1)=u_{B}(1)$. The optimum is then standardization on either $A$ or $B$.

19. Condition T4 requires that the second derivatives (left term) are not too big compared to the first derivatives (right term). The linear case, which is both convex and concave, is an extreme example of weak concavity which fits with condition T4. An example of strictly concave functions satisfying assumptions A1 and T4 is: $u_{A}(x)=20 x-x^{2}$ and $u_{B}(y)=0.5\left(20 y-y^{2}\right)$. 
20. The concavity of the utility functions implies that the slope of $u_{k}(x)$ is always smaller than the slope of the line connecting $\left(0, u_{k}(0)\right)$ to $\left(x, u_{k}(x)\right)$, that is $\left.\left.u_{k}^{\prime}(x) \leq \frac{u_{k}(x)}{x} \forall x \in\right] 0,1\right](k=A, B)$. This implies $u_{A}^{\prime}(1) \leq u_{A}(1)$ and $u_{B}^{\prime}(1) \leq u_{B}(1)$ such that $u_{A}^{\prime}(1)>u_{B}^{\prime}(0)$ $\Rightarrow u_{A}(1)>u_{B}^{\prime}(0)$ and $u_{B}^{\prime}(1)>u_{A}^{\prime}(0) \Rightarrow u_{B}(1)>u_{A}^{\prime}(0)$. Condition $\mathrm{T} 2+$ (respectively $\mathrm{T} 3+$ ) is stronger than condition T2 (respectively T3). They are equivalent in the linear case.

21. For instance, if the utility functions increase to a certain market share ceiling and then stagnate, even with one standard inferior to the other (e.g. under $T 2$ but not $u_{A}^{\prime}(1)>u_{B}^{\prime}(0)$ ), standardization is sub-optimal.

22. A study in Nigeria concluded that up to $60 \%$ of medicines on the street market were counterfeit. 

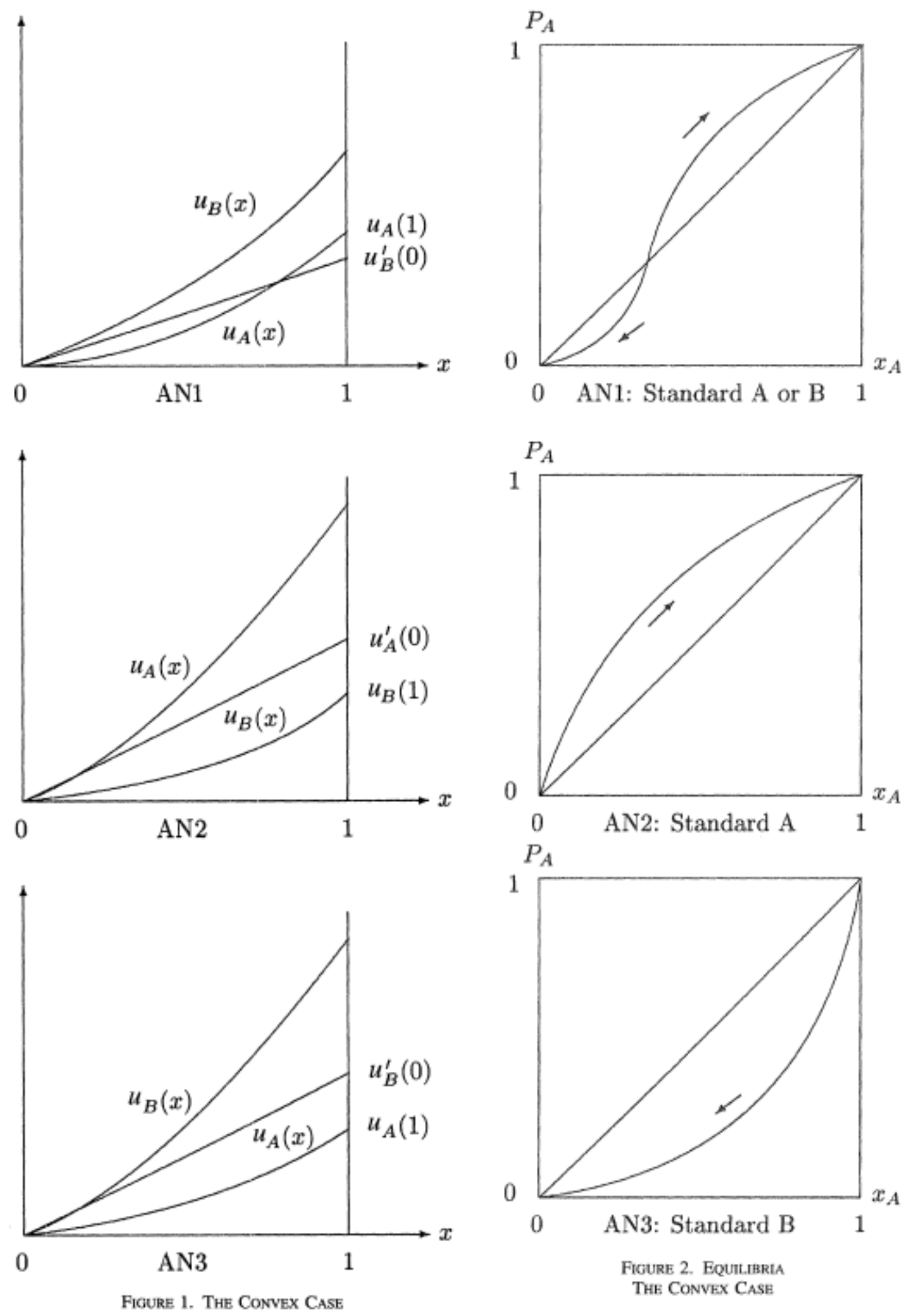

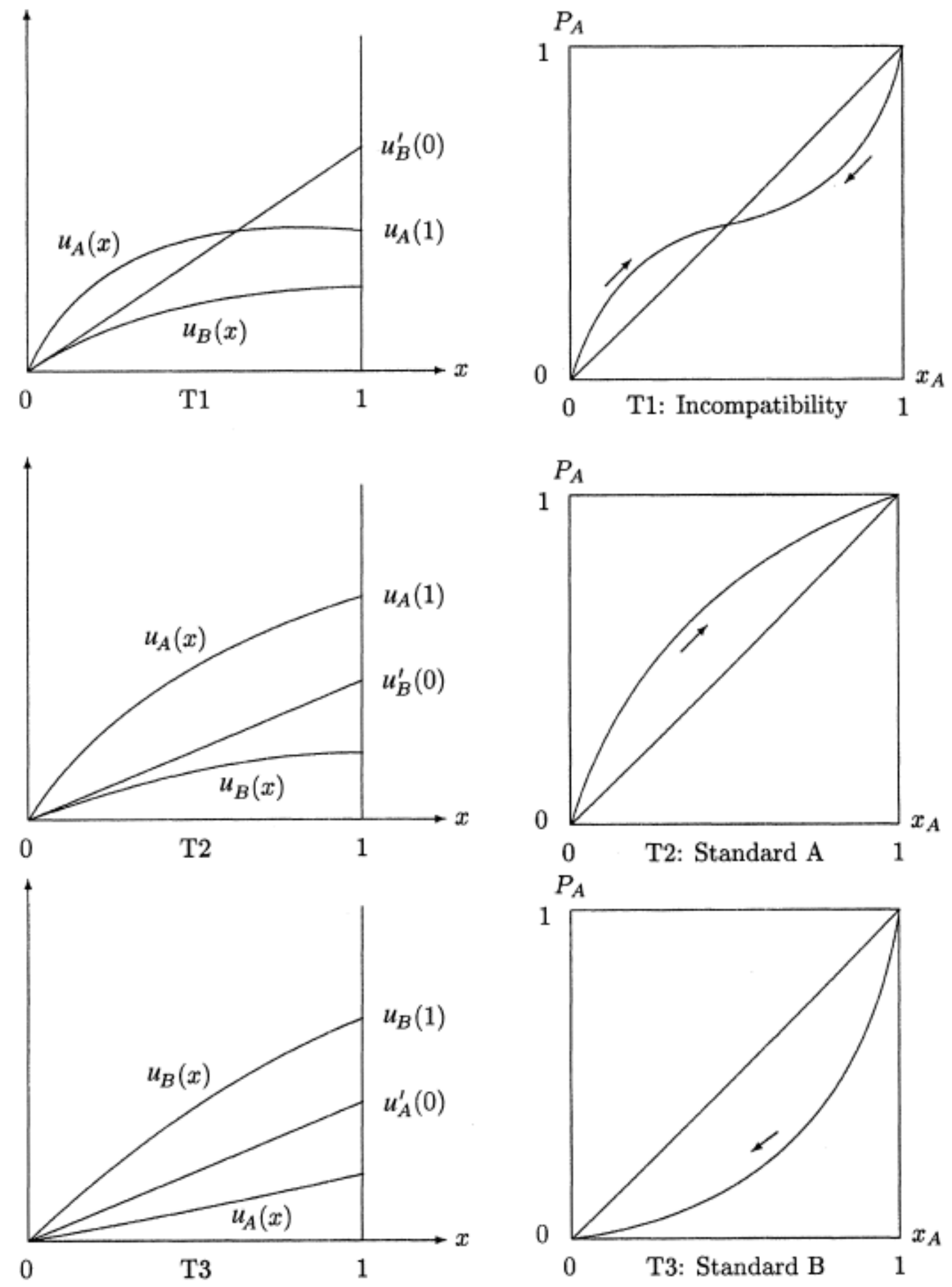
$P_{A}$

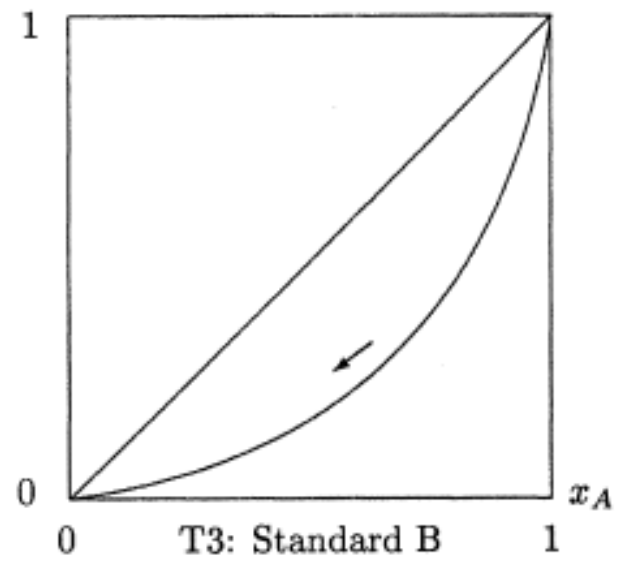

Figure 3. The Concave Case

Figure 4. EquLlibria

The Concave Case 


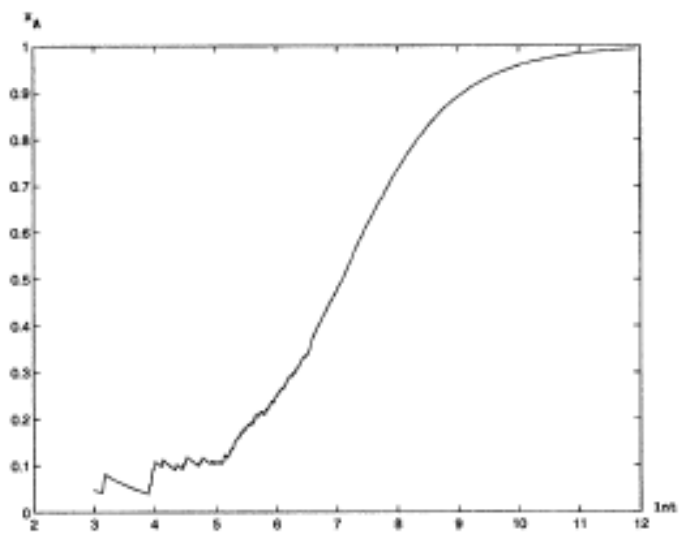

a. Convex Case

$u_{A}(x)=(1+x)^{1,5}-1 \quad u_{B}(x)=x^{2}$

$N_{A}^{0}=1 \quad N_{b}^{0}=20 \quad T=150,000$

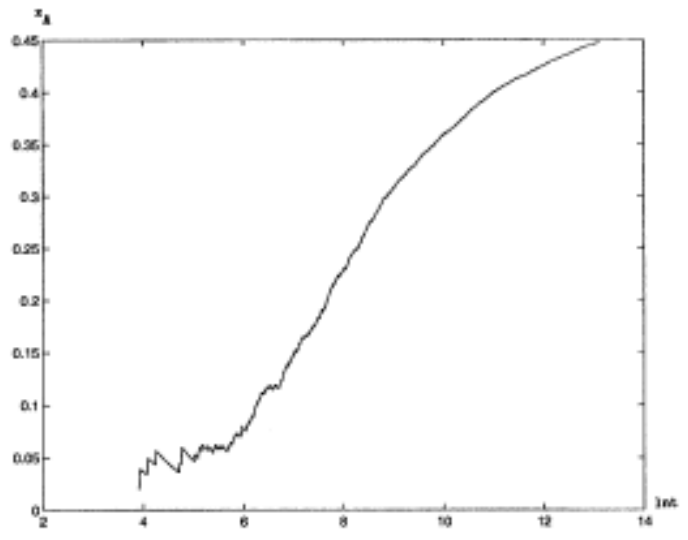

c. Concave Case (Incompatiblity Equilibrium)

$$
u_{A}(x)=u_{B}(x)=x(2-x)
$$$$
N_{A}^{0}=1 \quad N_{b}^{0}=50 \quad T=500,000
$$

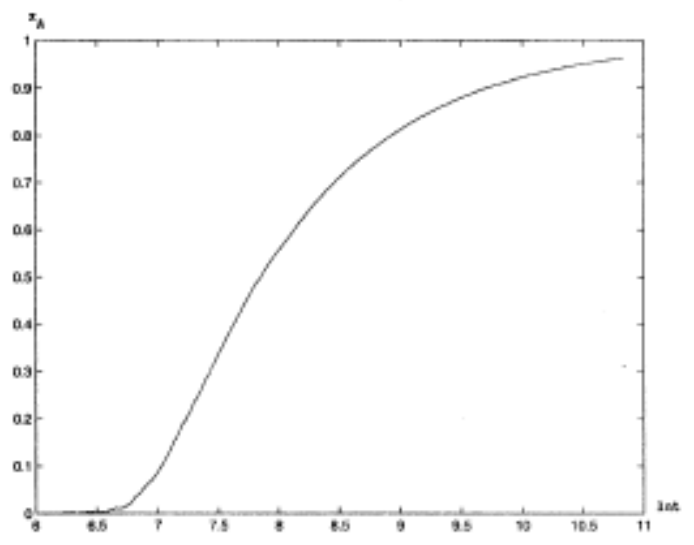

b. Linear Case

$u_{A}(x)=10 x \quad u_{B}(x)=x$

$N_{A}^{0}=1 \quad N_{g}^{0}=500 \quad T=50,000$

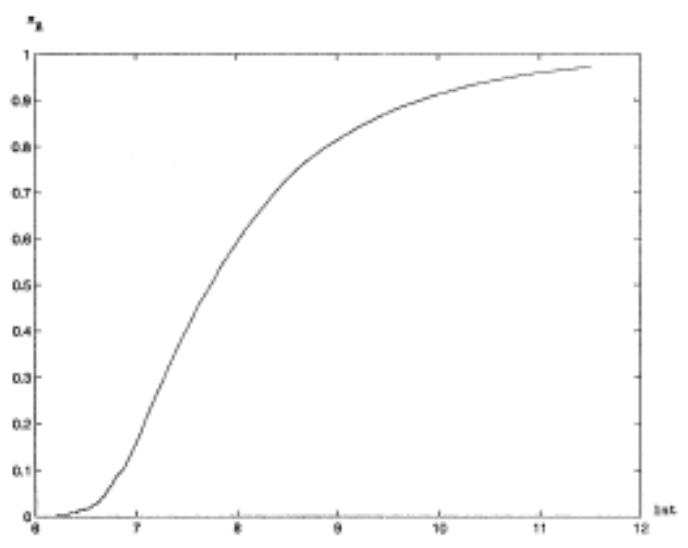

d. Concave Case (Standardization) $u_{A}(x)=x(10-x) \quad u_{H}(x)=x(2-x$

$N_{A}^{0}=1 \quad N_{B}^{0}=500 \quad T=100,000$

Figure 5. Diffusion Curves 